\title{
Association between aberrant APC promoter methylation and breast cancer pathogenesis: a meta-analysis of 35 observational studies
}

\author{
Dan Zhou ${ }^{1,2}$, Weiwei Tang ${ }^{3}$, Wenyi Wang ${ }^{3}$, Xiaoyan Pan ${ }^{3}$, Han-Xiang An Corresp., 3 , Yun Zhang ${ }^{\text {Corresp. } 1,2}$ \\ 1 Department of Translational medicine, Xiamen Institute of Rare Earth Materials, Xiamen, China \\ 2 Department of Translational medicine, Key Laboratory of Design and Assembly of Functional Nanostructures, Fujian Provincial Key Laboratory of \\ Nanomaterials, Fujian Institute of Research on the Structure of Matter, Fuzhou, China \\ 3 Department of Medical Oncology, The First Affiliated Hospital of Xiamen University, Xiamen, China \\ Corresponding Authors: Han-Xiang An, Yun Zhang \\ Email address: anhanxiang@yahoo.com, zhangy@fjirsm.ac.cn
}

Background: Adenomatous polyposis coli (APC) is widely known as an antagonist of the Wnt signaling pathway via the inactivation of $\beta$-catenin. An increasing number of studies have reported that APC methylation contributes to the predisposition to breast cancer (BC). However, recent studies have yielded conflicting results. Methods: Herein, we systematically carried out a meta-analysis to assess the correlation between APC methylation and BC risk. Based on searches of the Cochrane Library, PubMed, Web of Science and Embase databases, the odds ratio (OR) with 95\% confidence interval (Cl) values were pooled and summarized. Results: A total of 31 articles involving 35 observational studies with 2482 cases and 1212 controls met the inclusion criteria. The results demonstrated that the frequency of APC methylation was significantly higher in BC cases than controls under a random effect model $(\mathrm{OR}=9.97,95 \% \mathrm{Cl}=5.66-17.57)$. Subgroup analysis further confirmed the reliable results, regardless of the sample types detected, methylation detection methods applied and different regions included. Interestingly, our results also showed that the frequency of APC methylation was significantly lower in early-stage $B C$ patients than late-stage ones $(\mathrm{OR}=0.62,95 \% \mathrm{Cl}=0.42$ 0.93). Conclusion: APC methylation might play an indispensable role in the pathogenesis of $\mathrm{BC}$ and could be regarded as a potential biomarker for the diagnosis of BC. 
1 Association between aberrant APC promoter methylation and breast cancer pathogenesis:

2 a meta-analysis of 35 observational studies

3 Dan Zhou ${ }^{1,2}$, Weiwei Tang 3 , Wenyi Wang ${ }^{3}$, Xiaoyan Pan ${ }^{3}$, Han-Xiang $\mathrm{An}^{3,4}$ and Yun Zhang ${ }^{1,2,4}$

$4{ }^{1}$ Department of Translational medicine, Xiamen Institute of Rare Earth Materials, Chinese

5 Academy of Sciences, Xiamen, 361021, China

$6 \quad{ }^{2}$ Department of Translational medicine, Key Laboratory of Design and Assembly of Functional

7 Nanostructures, Fujian Provincial Key Laboratory of Nanomaterials, Fujian Institute of Research

8 on the Structure of Matter, 350002, Chinese Academy of Sciences, China

$9{ }^{3}$ Department of Medical Oncology, The First Affiliated Hospital of Xiamen University, Xiamen,

10 Fujian, 361001, China

$11{ }^{4}$ Each of the following is a corresponding author: Yun Zhang and Han-Xiang An. Yun Zhang,

12 Xiamen Institute of Rare Earth Materials, Chinese Academy of Sciences, 17th Floor,

13 Technovation Build, Ji-mei Rd, Xiamen, Fujian, 361021, China; Email: zhangy@fjirsm.ac.cn

Han-Xiang An, Department of Medical Oncology, The First Affiliated Hospital of Xiamen

University, Xiamen, Fujian, 361001, China; Email: anhanxiang@yahoo.com 
18 Background: Adenomatous polyposis coli (APC) is widely known as an antagonist of the Wnt

19 signaling pathway via the inactivation of $\beta$-catenin. An increasing number of studies have reported that APC methylation contributes to the predisposition to breast cancer (BC). However, recent studies have yielded conflicting results.

Methods: Herein, we systematically carried out a meta-analysis to assess the correlation between APC methylation and BC risk. Based on searches of the Cochrane Library, PubMed, Web of Science and Embase databases, the odds ratio (OR) with 95\% confidence interval (CI) values were pooled and summarized.

Results: A total of 31 articles involving 35 observational studies with 2482 cases and 1212 controls met the inclusion criteria. The results demonstrated that the frequency of APC methylation was significantly higher in BC cases than controls under a random effect model $(\mathrm{OR}=9.97,95 \% \mathrm{CI}=5.66-17.57)$. Subgroup analysis further confirmed the reliable results, regardless of the sample types detected, methylation detection methods applied and different regions included. Interestingly, our results also showed that the frequency of APC methylation was significantly lower in early-stage $\mathrm{BC}$ patients than late-stage ones $(\mathrm{OR}=0.62,95 \% \mathrm{CI}=0.42$ $0.93)$.

Conclusion: APC methylation might play an indispensable role in the pathogenesis of BC and could be regarded as a potential biomarker for the diagnosis of $\mathrm{BC}$. 
Introduction

41 Breast cancer (BC) is the most common malignancy and the leading cause of cancer death 42 among females in both well and poorly developed countries, accounting for approximately $15 \%$ 43 of all cancer deaths in 2012 (Torre et al. 2015). It is well established that BC is a clinically and 44 pathologically heterogeneous disease and has been categorized into five subtypes (i.e., luminal A and $\mathrm{B}$, human epidermal growth receptor-2, triple negative and basal-like) based on various biological markers (Inoue \& Fry 2015). Risk factors including reproductive, hormonal and environmental factors, have been associated with an increased incidence of BC (Harrison et al. 2015). Previous studies have reported that early detection using mammography is effective and can improve the overall survival rate (Brooks et al. 2010). However, false positive mammograms always result in the over-diagnosis and over-treatment of developing $\mathrm{BC}$. Therefore, no acknowledged biomarker has yet been proven to be sufficiently sensitive and specific for routine use in clinical diagnosis.

Epigenetic as well as genetic alterations are both stable and heritable and occur in tumor suppressor genes involved in tumourigenesis. The most common epigenetic alteration involving aberrant DNA methylation, a reliable and sensitive biomarker for nearly all types of cancer including breast cancer, often leads to the transcriptional silencing of tumor suppressor genes

57 (Zmetakova et al. 2013). Several studies have demonstrated that tumor DNA derived from malignant cells can be detected in various bodily fluids and serum of BC patients and can potentially serve as a non-invasive diagnostic material (Martínez-Galán et al. 2014). A growing 
60

61

62

63

64

65

66

67

number of tumor suppressor genes has been shown to be directly involved in cell cycle regulation, DNA repair, cell signal transduction and angiogenesis (Dumitrescu 2012). Notably, the promoter methylation of genes involved in the canonical Wnt signaling pathway, which regulates cell differentiation, proliferation and homeostasis, are observed more often in $\mathrm{BC}$ patients compared with cancer-free controls (Klarmann et al. 2014).

The adenomatous polyposis coli (APC) gene is widely known as an antagonist of the Wnt signaling pathway via the inactivation of $\beta$-catenin, which is regarded as a transcriptional activator (Virmani et al. 2001). The APC gene, located at chromosome 5q21-5q22, was originally implicated in colorectal cancer (Van der Auwera et al. 2008). The inhibition or downregulation of APC expression through APC promoter methylation contributes to the formation of colorectal cancer (Ashktorab et al. 2013). Similar to the findings in colorectal cancer, APC promoter methylation is associated with various early- or late-stage human malignancies, including BC (Matsuda et al. 2009). The promoter hypermethylation of APC is most often related to the nuclear accumulation of $\beta$-catenin, which may result in the loss of cell growth control (Sparks et al. 1998). Thus, APC promoter methylation, which acts as a non-invasive biomarker, can be used to distinguish $\mathrm{BC}$ patients from cancer-free controls. However, recent studies have yielded conflicting results with regard to the significant association between APC methylation and $\mathrm{BC}$ pathogenesis. Wojdacz $\mathrm{KT}$ et al. reported that there was no significant difference in the frequency of APC methylation in peripheral blood leukocyte DNA between BC patients and cancer-free controls(Wojdacz et al. 2011a). CHO YH et al. also showed that the APC gene was rarely hypermethylated in blood DNA in BC patients (Cho et al. 2010). 
81 Given these controversial results, we conducted this comprehensive meta-analysis of the

82

83

84

85 current observational studies to evaluate the association between the aberrant methylation of the APC promoter and increased BC risk.

\section{Materials \& Methods}

\section{Search Strategy}

Eligible studies were identified by searching the following databases until February 2016: the Cochrane Library, PubMed, Web of Science and Embase. No language restrictions or lower data limits were imposed; only abstracts, unpublished and incomplete studies were excluded. Titles, abstracts of potential references and reference lists from relevant studies were carefully checked. We performed the search strategy using the following search terms and their various combinations: "APC," "Adenomatous polyposis coli," "methylation," "breast cancer," "breast neoplasm" and "mammary carcinoma."

\section{Selection Criteria}

The studies included in the present meta-analysis addressed the association between APC methylation and increased BC risk. Our inclusion criteria were as follows: (1) provided sufficient data on the frequency of APC methylation in BC patients and controls; (2) original observational studies in full-text form; and (3) when several studies overlapped, the most recent or large-scale article was selected. The following were exclusion criteria: (1) data based on reviews, animal models, case reports or cell line studies; (2) studies lacking key information necessary for calculations; (3) duplicated studies; and (4) studies including BC patients or controls who underwent radiotherapy and chemotherapy which may influence APC promoter methylation 
102 levels.

\section{Data extraction}

104 The relevant data were extracted from the eligible studies independently by two authors (D 105 Zhou and WW Tang). Differing opinions, if any, were resolved by discussion in accordance with 106 the original literature. The following information was extracted in a predefined table: the name 107 of the first author, the year of publication, the country of origin, the sample type, the experimental methods used to detect APC methylation, sample size, tumor stage, tumor grade and APC methylation frequencies. Additionally, we classified stage 0, I and II as early-stage BC and stage III and IV as late-stage BC, as confirmed by the AJCC staging system. Furthermore, grades I and II were combined as low-grade BC; grade III was regarded as high-grade BC. This meta-analysis was performed following the statement of preferred reporting items set by the

PRISMA Group (File S1) (Moher et al. 2009).

\section{Statistical analysis}

All analyses were carried out using Review Manager 5.3 (Cochrane Collaboration) and Stata effects according to the $95 \%$ confidence interval (CI). The association between APC promoter methylation and $\mathrm{BC}$ risk was evaluated by calculating the odds ratio (OR) with corresponding 95\%CI. For individual studies the OR was represented by a square and the $95 \% \mathrm{CI}$ by a horizontal line in the centre of the forest plot. The OR and associated $95 \% \mathrm{CIs}$ in the centre of the forest plot were plotted on a logarithmic scale. When a CI did not include 1.0, a correlation was deemed statistically significant. Heterogeneity between the included studies was quantified 
123 through Q-tests based on the chi-square test and $\mathrm{I}^{2}$ value. An $\mathrm{I}^{2}$ value $>50 \%$ and a $\mathrm{p}<0.10$ denoted

124 strong heterogeneity, an $\mathrm{I}^{2}$ value $=25-50 \%$ denoted a moderate degree of heterogeneity and an $\mathrm{I}^{2}$

125 value $<25 \%$ or a $\mathrm{p}>0.10$ denoted mild heterogeneity (Higgins et al. 2003). A random effect

126 model was used when statistical heterogeneity existed among studies $(\mathrm{p}<0.1)$. Otherwise, the

127 fixed effect model was employed (Li et al. 2014). Moreover, the subgroup meta-analyses were

128 also performed according to region, experimental methods for detecting APC methylation, and

129 sample types in order to explore the potential origin of inter-study heterogeneity. In addition, we

130 conducted a sensitivity analysis by removing a single study to examine the stability of the results.

131 The funnel plot, Begg's test and Egger's test were investigated in order to determine the degree

132 of publication bias. The treatment effect was plotted against a measure of study size in the funnel

133 plot. When publication bias was present, the shape of the funnel plot was asymmetric. Trim and

134 fill analysis was used to estimate the number of potential missing studies resulting from the

135 asymmetry of the funnel plot.

\section{Results}

\section{Study selection and Characteristics}

138 The selection process is displayed as a flow chart in Fig. 1 based on the search strategies as

previously described. After a careful initial search of the abstracts, 74 potentially relevant articles

were identified excluding 1 duplicate and 93 irrelevant studies. Then, we reviewed the full text

articles. Among these studies, 43 were excluded (21 articles did not design a control group; 9

articles focused on BC cell lines; 8 articles lacked available data; and 5 articles were reviews).

Finally, 31 studies published from 2001 to 2016 involving 35 studies were included in this 
144 systematic meta-analysis (PubMed 19, Web of Science 10, Embase 2).

145 The general characteristics of eligible studies were summarized and displayed in Table 1. A 146 total of $2482 \mathrm{BC}$ patients and 1212 controls were employed in multiple countries or regions 147 including Asia (n=10) (Jin et al. 2001; Jing et al. 2010; Jung et al. 2013; Lee et al. 2004; Liu et al. 148 2007; Park et al. 2011b; Prasad et al. 2008; Zhang et al. 2007), Europe (n=13) (Fridrichova et al. 149 2015; Hoque et al. 2009; Jeronimo et al. 2008; Martins et al. 2011; Matuschek et al. 2010; 150 Muller et al. 2003; Parrella et al. 2004; Rykova et al. 2004; Van der Auwera et al. 2009a; Van 151 der Auwera et al. 2009b; Van der Auwera et al. 2008; Wojdacz et al. 2011b), Africa (n=2) 152 (Hoque et al. 2006; Swellam et al. 2015), North America (n=9) (Brooks et al. 2010; Chen et al. 153 2011; Cho et al. 2010; Dulaimi et al. 2004; Lewis et al. 2005; Shinozaki et al. 2005; Taback et al. 154 2006; Virmani et al. 2001) and Oceania (n=1) (Pang et al. 2014). Furthermore, the methylated 155 APC levels in BC patients and controls were examined with 6 methods. Of these methods, 156 methylation specific PCR (MSP) was adopted in 17 studies, quantitative real-time MSP (QMSP) 157 was used in 9 studies, methylation specific-multiplex ligation-dependent probe amplification 158 (MethyLight) was used in 4 studies, methylation specific-multiplex ligation-dependent probe 159 amplification (MS-MLPA) was employed in 2 studies, methylation-sensitive high-resolution 160 melting analysis (MS-HRM) was used in 2 studies and pyrosequencing was used in only 1 study.

161 Furthermore, BC tissues (i.e., fresh frozen tissues, formalin fixed paraffin-embedded tissues and 162 tissues from surgery), samples derived from blood (i.e., blood cells and serum) and needle 163 aspirated fluid (NAF) were enrolled to assess the methylation levels of the APC promoter. 
165

166

167

168

169

170

171

172

173

174

175

176

177

178

179

180

181

182

183

184

185

The pooled results of this meta-analysis reflected the association between APC promoter

methylation and BC pathogenesis (Fig. 2). Due to the existence of significant heterogeneity among the included studies $\left(\mathrm{p}<0.00001, \mathrm{I}^{2}=68 \%\right)$, the random effect model was adopted to evaluate the combined effects of APC promoter methylation. The overall analysis indicated that the frequency of APC promoter methylation was remarkably higher in BC patients than in cancer-free controls. The combined OR for 35 included relevant studies showed that APC methylation was significantly correlated with increased $\mathrm{BC}$ risk and the absence of APC expression played an important role in $\mathrm{BC}$ pathogenesis $(\mathrm{OR}=9.97,95 \% \mathrm{CI}=5.66-17.57)$.

\section{Sensitivity analysis}

A sensitivity analysis was conducted by omitting one individual study every time to evaluate the stability of the pooled OR and to choose the heterogeneous study. As shown in Fig. 3, the combined OR between APC methylation and increased BC risk was indeed reliable without heterogeneous studies.

\section{Subgroup analysis}

Due to the significant existence of inter-study heterogeneity $\left(\mathrm{p}<0.00001, \mathrm{I}^{2}=68 \%\right)$, subgroup analysis based on region, experimental methods for the detection of APC methylation and sample types were carried out to appraise the sources of the heterogeneity (Table 2). With regard to subgroup analysis based on region, heterogeneity in Asian subgroups disappeared completely $\left(\mathrm{I}^{2}=0 \%\right)$ and the pooled $\mathrm{OR}$ value was $27.21[13.51,54.81]$. The $\mathrm{I}^{2}$ value representing heterogeneity in the European and North American subgroups decreased by 8\% and 26\%, compared with the overall $\mathrm{I}^{2}$ value. Furthermore, their OR values also decreased to 5.50[2.69, 
186 187 188 189 190 191 192 193 194 195 196 197 198 199 200 201 202 203 204 205 206

11.22] and 3.79[1.70, 8.45]. In the African subgroup, the OR was $172.05[1.76,16792.96]$ with higher heterogeneity $\left(\mathrm{I}^{2}=80 \%\right)$ due to the small subset containing only 2 studies. These results indicated that the heterogeneity might result from different regions and APC methylation was remarkably related to increased $\mathrm{BC}$ risk without geographical restrictions. For the subgroup analyses based on sample types, the blood or serum group $(\mathrm{OR}=11.79,95 \% \mathrm{CI}=3.02-46.06)$ made the largest contribution to the heterogeneity $\left(\mathrm{I}^{2}=80 \%\right)$. In the tissue subgroup, the OR was $10.68[5.50,20.75]$ with lower heterogeneity $\left(\mathrm{I}^{2}=53 \%\right)$. Moreover, heterogeneity in the NAF subgroups could be ignored $\left(\mathrm{I}^{2}=6 \%\right)$. These results confirmed the stable association between APC methylation and BC risk in different sample types. For studies based on the methods used to detect the methylation of the APC promoter, the combined OR value was 18.18 for MSP $(95 \% \mathrm{CI}=7.96-41.52), 3.92$ for QMSP $(95 \% \mathrm{CI}=1.77-8.70), 8.05$ for Methylight $(95 \% \mathrm{CI}=1.50$ 43.12) and 31.81 for MS-MLPA $(95 \% \mathrm{CI}=5.30-191.06)$. Heterogeneity in the QMSP $\left(\mathrm{I}^{2}=40 \%\right)$ and MS-MLPA $\left(\mathrm{I}^{2}=0 \%\right)$ subgroups was far lower than that of the MethyLight and MS-HRM subgroups $\left(\mathrm{I}^{2}=83 \%\right)$.

To assess the association between APC methylation and tumor stage, 11 studies comprising $681 \mathrm{BC}$ patients were pooled to calculate the OR. The results showed that the frequency of APC promoter methylation was significantly lower in early-stage patients than in late-stage patients $\left(\mathrm{OR}=0.62,95 \% \mathrm{CI}=0.42-0.93, \mathrm{I}^{2}=34 \%\right)$. Meanwhile, the $\mathrm{OR}$ of 8 studies revealed that the association between APC methylation and tumor grade was not statistically significant $(\mathrm{OR}=0.78$, $\left.95 \% \mathrm{CI}=0.51-1.21, \mathrm{I}^{2}=0 \%\right)$.

\section{Publication bias}


207 We used the funnel plot, Begg's test and Egger's test to evaluate the degree of publication bias.

208 The shape of the funnel plot had no obvious asymmetry (Fig. 4A). Moreover, Begg's test $209(\operatorname{Pr}>|z|=0.196>0.05)$ suggested no significant publication bias (Fig. 4B). Interestingly, Egger's test revealed evident statistical proof for the existence of publication bias $(\mathrm{p}>|\mathrm{t}|=0.000<0.05)$.

Therefore, we carried out trim and fill analysis to identify and revise the bias. As shown in Fig. 4C, 13 adjusted studies were added to the initial meta-analysis. The corrected OR was still highly significant for the association between APC methylation and $\mathrm{BC}$ risk $(\mathrm{OR}=1.621,95 \% \mathrm{CI}=1.081$ -

2.144), further proving the stability of our meta-analysis.

\section{Discussion}

To the best of our knowledge, this is the first meta-analysis to systematically evaluate the association between APC promoter methylation and BC pathogenesis. BC is a significant clinical and public health problem and is mainly attributed to epigenetic and genetic changes. Epigenetic alternation involving DNA methylation is a relatively early event that serves as a tumor molecular biomarker candidate in BC and can be detected in all pathological tumor stages. The

APC gene is considered to be a tumor suppressor gene, and the silencing of its expression may result in cell-to-cell adhesion disorders and the disruption of the Wnt signaling pathway. APC methylation, a contributing factor to the absence of APC expression, is often linked to $\beta$-catenin accumulation and TCF/LEF-induced transcription(Klarmann et al. 2008). Numerous studies have reported that APC methylation is highly specific for BC and can be used as a biomarker in the diagnosis of BC (Dumitrescu 2012; Van der Auwera et al. 2008). De-Pu Zhang et al. found that 
228

229

230

231

232

233

234

235

236

237

with breast cancer(Zhang et al. 2015). However, the role of APC methylation in BC pathogenesis remains controversial.

To resolve these contradictory results, we gathered relevant studies and carried out this metaanalysis using systematic statistical methods. Herein, we included a total of 35 studies with 2482 cases and 1212 controls published from 2001 to 2016. Our results based on the pooled OR revealed that the level of APC methylation was observably higher in $\mathrm{BC}$ patients compared to cancer-free controls, which indicated that APC methylation could serve as a potential biomarker for $\mathrm{BC}$ diagnosis, regardless of the various sample types detected, APC methylation detection methods applied and cases employed in different regions.

Then, we conducted subgroup analysis to identify the sources of the heterogeneity and found that various sample types, methylation detection methods and cases employed in different regions all contributed to the heterogeneity. In subgroup analysis based on sample types, the results showed that APC methylation was significantly related to $\mathrm{BC}$ pathogenesis, whether in tissue, blood or serum and NAF. Cell-free DNA in serum and plasma, which mostly originates from tumor cell degradation, can be collected and examined for epigenetic alterations with various malignancies (Anker et al. 1999). The sample materials including blood or serum, used for extracting DNA are often stored for different time periods which will produce false positives and false negatives. Thus, blood samples should be examined as rapidly as possible after being collected. Therefore, the accuracy of cell-free DNA largely depends on the standardized storage conditions. NAF is a rapid, minimally invasive and cheap diagnostic means with high sensitivity.

The accuracy of NAF mainly relies on the experience of the cytopathologist which may result in 
249 an increasing trend for false negatives (Jeronimo et al. 2003). In subgroup analysis based on

250 methylation detection methods, significant associations were observed when examined using

251 MSP, QMSP, MethyLight and MS-MLPA, except for MS-HRM. Among these, the pooled OR

252 derived from studies using MS-MLPA was the maximum with no heterogeneity. The diagnostic

253 accuracy of MS-MLPA was not affected by sample types (Park et al. 2011a). Cut-off values and

254 primers based on different CPG islands which were used in different studies, contributed to the

255 heterogeneity of other methods. In subgroup analysis based on different regions, APC

256 methylation was significantly correlated with BC patients in all included regions. The results

257 indicated that although the genetic factors, environments and life styles were totally different, the

258 correlation was still strong and stable. Therefore, an appropriate APC methylation detection

259 method considering the regions and sample types employed is essential for routine clinical

260 diagnosis. Additionally, we found that the status of APC methylation increased notably in late-

261 stage patients compared with early-stage ones, which indicated that APC methylation might be

262 closely related to the malignant evolution of BC.

263 As mentioned above, Wojdacz TK et al. examined the use of methylation biomarkers as

264 screening tools for BC diagnosis (Wojdacz et al. 2011b). They found no significant difference in

265 the frequency between $180 \mathrm{BC}$ patients and 108 healthy controls and a weak association between

266 APC methylation and BC pathogenesis. This discrepancy mainly resulted from the methylation

267 detection method. They used MS-HRM which may yield heterogeneous methylation values

268 derived from the primer and cut-off values, and it tended to produce a lower evaluation of

methylation when applying less methylated samples (Migheli et al. 2013). 
Surprisingly, only Egger's linear regression showed an obvious publication bias other than

Begg's test and funnel plots. Egger M et al. suggested that Egger's test was more sensitive than

Begg's test (Egger et al. 1997). The publication bias mainly resulted from the inclusion criteria.

Only full-text published studies were collected in this meta-analysis. Therefore, unpublished

studies and conference abstracts were not included. Additionally, other study characteristics including the source of funding and prevailing theories at the time of publication, can contribute to publication bias. However, we included a large number of BC patients $(n=2482)$ to ensure the reliability of the meta-analysis and minimize the potential publication bias.

Although the meta-analysis indeed confirmed the significance of a correlation between APC methylation and $\mathrm{BC}$ pathogenesis, several limitations should be considered. First, the sample sizes used in several studies were small, which may have increased the risk of publication bias and limited the results of the meta-analysis. Second, the quality of the selected studies varied, as we included high-quality and low-quality studies. Therefore, heterogeneity likely existed. Third, the cut-off points of APC methylation and the primers based on CPG islands were difficult to unify. Thus, we were unable to calculate the pooled sensitivity and specificity of APC methylation.

In conclusion, the results of our meta-analysis highlight the clinical significance and scientific value of APC promoter methylation in the diagnosis of BC. Consequently, APC methylation is a potential biomarker for monitoring $\mathrm{BC}$ development. However, given the limitations listed above, high-quality studies with large-scale and consistent standards should be carried out. The 
291

292

293

294

295

296

297

298

299

300

301

302

303

304

305

306

307

308

309

310

311

Institute are necessary for adaptation to high-quality studies(McShane et al. 2005).

\section{References}

Anker P, Mulcahy H, Chen XQ, and Stroun M. 1999. Detection of circulating tumour DNA in the blood (plasma/serum) of cancer patients. Cancer Metastasis Rev 18:65-73.

Ashktorab H, Rahi H, Wansley D, Varma S, Shokrani B, Lee E, Daremipouran M, Laiyemo A, Goel A, Carethers JM, and Brim H. 2013. Toward a comprehensive and systematic methylome signature in colorectal cancers. Epigenetics 8:807-815. 10.4161/epi.25497

Brooks JD, Cairns P, Shore RE, Klein CB, Wirgin I, Afanasyeva Y, and Zeleniuch-Jacquotte A. 2010. DNA methylation in pre-diagnostic serum samples of breast cancer cases: results of a nested case-control study. Cancer Epidemiol 34:717-723. 10.1016/j.canep.2010.05.006

Chen KM, Stephen JK, Raju U, and Worsham MJ. 2011. Delineating an epigenetic continuum for initiation, transformation and progression to breast cancer. Cancers (Basel) 3:1580-1592. $10.3390 /$ cancers 3021580

Cho YH, Yazici H, Wu HC, Terry MB, Gonzalez K, Qu M, Dalay N, and Santella RM. 2010. Aberrant promoter hypermethylation and genomic hypomethylation in tumor, adjacent normal tissues and blood from breast cancer patients. Anticancer Res 30:2489-2496.

Dulaimi E, Hillinck J, Ibanez de Caceres I, Al-Saleem T, and Cairns P. 2004. Tumor suppressor gene promoter hypermethylation in serum of breast cancer patients. Clin Cancer Res 10:6189-

6193. 10.1158/1078-0432.CCR-04-0597

Dumitrescu RG. 2012. Epigenetic markers of early tumor development. Methods Mol Biol 
313 Egger M, Davey Smith G, Schneider M, and Minder C. 1997. Bias in meta-analysis detected by

314 a simple, graphical test. BMJ 315:629-634.

315 Fridrichova I, Smolkova B, Kajabova V, Zmetakova I, Krivulcik T, Mego M, Cierna Z, Karaba 316 M, Benca J, Pindak D, Bohac M, Repiska V, and Danihel L. 2015. CXCL12 and ADAM23

317 hypermethylation are associated with advanced breast cancers. Transl Res 165:717-730. $318 \quad 10.1016 / \mathrm{j} . \operatorname{trs} 1.2014 .12 .006$

319 Harrison K, Hoad G, Scott P, Simpson L, Horgan GW, Smyth E, Heys SD, and Haggarty P. 2015. Breast cancer risk and imprinting methylation in blood. Clin Epigenetics 7:92. $10.1186 / \mathrm{s} 13148-015-0125-\mathrm{x}$

Higgins JP, Thompson SG, Deeks JJ, and Altman DG. 2003. Measuring inconsistency in metaanalyses. BMJ 327:557-560. 10.1136/bmj.327.7414.557

Hoque MO, Feng Q, Toure P, Dem A, Critchlow CW, Hawes SE, Wood T, Jeronimo C,

Rosenbaum E, Stern J, Yu M, Trink B, Kiviat NB, and Sidransky D. 2006. Detection of aberrant methylation of four genes in plasma DNA for the detection of breast cancer. $J$ Clin Oncol 24:4262-4269. 10.1200/JCO.2005.01.3516

Hoque MO, Prencipe M, Poeta ML, Barbano R, Valori VM, Copetti M, Gallo AP, Brait M,

Maiello E, Apicella A, Rossiello R, Zito F, Stefania T, Paradiso A, Carella M, Dallapiccola B, Murgo R, Carosi I, Bisceglia M, Fazio VM, Sidransky D, and Parrella P. 2009. Changes in CpG islands promoter methylation patterns during ductal breast carcinoma progression. Cancer 
333 Inoue K, and Fry EA. 2015. Aberrant Splicing of Estrogen Receptor, HER2, and CD44 Genes in

334 Breast Cancer. Genet Epigenet 7:19-32. 10.4137/GEG.S35500

335 Jeronimo C, Costa I, Martins MC, Monteiro P, Lisboa S, Palmeira C, Henrique R, Teixeira MR,

336 and Lopes C. 2003. Detection of gene promoter hypermethylation in fine needle washings from

337 breast lesions. Clin Cancer Res 9:3413-3417.

338 Jeronimo C, Monteiro P, Henrique R, Dinis-Ribeiro M, Costa I, Costa VL, Filipe L, Carvalho

339 AL, Hoque MO, Pais I, Leal C, Teixeira MR, and Sidransky D. 2008. Quantitative 340 hypermethylation of a small panel of genes augments the diagnostic accuracy in fine-needle 341 aspirate washings of breast lesions. Breast Cancer Res Treat 109:27-34. 10.1007/s10549-007$3429620-\mathrm{x}$

343 Jin Z, Tamura G, Tsuchiya T, Sakata K, Kashiwaba M, Osakabe M, and Motoyama T. 2001. 344 Adenomatous polyposis coli (APC) gene promoter hypermethylation in primary breast cancers. Br J Cancer 85:69-73. 10.1054/bjoc.2001.1853

346 Jing F, Yuping W, Yong C, Jie L, Jun L, Xuanbing T, and Lihua H. 2010. CpG island methylator 347 phenotype of multigene in serum of sporadic breast carcinoma. Tumour Biol 31:321-331. $10.1007 / \mathrm{s} 13277-010-0040-\mathrm{x}$

349 Jung EJ, Kim IS, Lee EY, Kang JE, Lee SM, Kim DC, Kim JY, and Park ST. 2013. Comparison 350 of methylation profiling in cancerous and their corresponding normal tissues from korean patients with breast cancer. Ann Lab Med 33:431-440. 10.3343/alm.2013.33.6.431

352 Klarmann GJ, Decker A, and Farrar WL. 2008. Epigenetic gene silencing in the Wnt pathway in 353 breast cancer. Epigenetics 3:59-63. 
354 Klarmann GJ, Decker A, and Farrar WL. 2014. Epigenetic gene silencing in the Wnt pathway in 355 breast cancer. Epigenetics 3:59-63. 10.4161/epi.3.2.5899

356 Lee A, Kim Y, Han K, Kang CS, Jeon HM, and Shim SI. 2004. Detection of Tumor Markers 357 Including Carcinoembryonic Antigen, APC, and Cyclin D2 in Fine-Needle Aspiration Fluid of 358 Breast. Arch Pathol Lab Med 128:1251-1256. $10.1043 / 1543-$ 2165(2004)128<1251:DOTMIC>2.0.CO;2

Lewis CM, Cler LR, Bu DW, Zochbauer-Muller S, Milchgrub S, Naftalis EZ, Leitch AM, Minna JD, and Euhus DM. 2005. Promoter hypermethylation in benign breast epithelium in relation to predicted breast cancer risk. Clin Cancer Res 11:166-172.

Li S, Zeng XT, Ruan XL, Weng H, Liu TZ, Wang X, Zhang C, Meng Z, and Wang XH. 2014. Holmium laser enucleation versus transurethral resection in patients with benign prostate hyperplasia: an updated systematic review with meta-analysis and trial sequential analysis. PLoS One 9:e101615. 10.1371/journal.pone.0101615

Liu Z, Yang L, Cui DX, Liu BL, Zhang XB, Ma WF, and Zhang Q. 2007. [Methylation status and protein expression of adenomatous polyposis coli (APC) gene in breast cancer]. Ai Zheng 26:586-590.

Martínez-Galán J, Torres B, del Moral R, Muñoz-Gámez JA, Martín-Oliva D, Villalobos M,

Núñez MI, Luna JdD, Oliver FJ, and Almodóvar JMRd. 2014. Quantitative detection of methylated ESR1 and 14-3-3- $\sigma$ gene promoters in serum as candidate biomarkers for diagnosis of breast cancer and evaluation of treatment efficacy. Cancer Biology \& Therapy 7:958-965. 
375 Martins AT, Monteiro P, Ramalho-Carvalho J, Costa VL, Dinis-Ribeiro M, Leal C, Henrique R, 376 and Jeronimo C. 2011. High RASSF1A promoter methylation levels are predictive of poor 377 prognosis in fine-needle aspirate washings of breast cancer lesions. Breast Cancer Res Treat 129:1-9. 10.1007/s10549-010-1160-0

Matsuda Y, Schlange T, Oakeley EJ, Boulay A, and Hynes NE. 2009. WNT signaling enhances breast cancer cell motility and blockade of the WNT pathway by sFRP1 suppresses MDA-MB231 xenograft growth. Breast Cancer Res 11:R32. 10.1186/bcr2317

Matuschek C, Bolke E, Lammering G, Gerber PA, Peiper M, Budach W, Taskin H, Prisack HB,

Schieren G, Orth K, and Bojar H. 2010. Methylated APC and GSTP1 genes in serum DNA correlate with the presence of circulating blood tumor cells and are associated with a more aggressive and advanced breast cancer disease. Eur J Med Res 15:277-286.

McShane LM, Altman DG, Sauerbrei W, Taube SE, Gion M, Clark GM, and Statistics

Subcommittee of the NCIEWGoCD. 2005. Reporting recommendations for tumor marker prognostic studies (REMARK). J Natl Cancer Inst 97:1180-1184. 10.1093/jnci/dji237

Migheli F, Stoccoro A, Coppede F, Wan Omar WA, Failli A, Consolini R, Seccia M, Spisni R,

Miccoli P, Mathers JC, and Migliore L. 2013. Comparison study of MS-HRM and pyrosequencing techniques for quantification of APC and CDKN2A gene methylation. PLoS One 8:e52501. 10.1371/journal.pone.0052501

Moher D, Liberati A, Tetzlaff J, Altman DG, and Group P. 2009. Preferred reporting items for systematic reviews and meta-analyses: the PRISMA statement. J Clin Epidemiol 62:1006-1012. 
396

397

398

399

400

401

402

403

404

405

406

407

408

409

410

411

412

413

414

415

416

Muller HM, Widschwendter A, Fiegl H, Ivarsson L, Goebel G, Perkmann E, Marth C, and

Widschwendter M. 2003. DNA methylation in serum of breast cancer patients: an independent prognostic marker. Cancer Res 63:7641-7645.

Pang JM, Deb S, Takano EA, Byrne DJ, Jene N, Boulghourjian A, Holliday A, Millar E, Lee CS, O'Toole SA, Dobrovic A, and Fox SB. 2014. Methylation profiling of ductal carcinoma in situ and its relationship to histopathological features. Breast Cancer Res 16:423. 10.1186/s13058014-0423-9

Park CK, Kim J, Yim SY, Lee AR, Han JH, Kim CY, Park SH, Kim TM, Lee SH, Choi SH, Kim SK, Kim DG, and Jung HW. 2011a. Usefulness of MS-MLPA for detection of MGMT promoter methylation in the evaluation of pseudoprogression in glioblastoma patients. Neuro Oncol 13:195-202. 10.1093/neuonc/noq162

Park SY, Kwon HJ, Lee HE, Ryu HS, Kim SW, Kim JH, Kim IA, Jung N, Cho NY, and Kang GH. 2011b. Promoter CpG island hypermethylation during breast cancer progression. Virchows Arch 458:73-84. 10.1007/s00428-010-1013-6

Parrella P, Poeta ML, Gallo AP, Prencipe M, Scintu M, Apicella A, Rossiello R, Liguoro G, Seripa D, Gravina C, Rabitti C, Rinaldi M, Nicol T, Tommasi S, Paradiso A, Schittulli F, Altomare V, and Fazio VM. 2004. Nonrandom distribution of aberrant promoter methylation of cancer-related genes in sporadic breast tumors. Clin Cancer Res 10:5349-5354. 10.1158/10780432.CCR-04-0555

Prasad CP, Mirza S, Sharma G, Prashad R, DattaGupta S, Rath G, and Ralhan R. 2008. Epigenetic alterations of CDH1 and APC genes: relationship with activation of $\mathrm{Wnt} /$ beta-catenin 
417 pathway in invasive ductal carcinoma of breast. Life Sci 83:318-325. 10.1016/j.1fs.2008.06.019

418 Rykova EY, Skvortsova TE, Laktionov PP, Tamkovich SN, Bryzgunova OE, Starikov AV, 419 Kuznetsova NP, Kolomiets SA, Sevostianova NV, and Vlassov VV. 2004. Investigation of 420 tumor-derived extracellular DNA in blood of cancer patients by methylation-specific PCR. Nucleosides Nucleotides Nucleic Acids 23:855-859. 10.1081/NCN-200026031

Shinozaki M, Hoon DS, Giuliano AE, Hansen NM, Wang HJ, Turner R, and Taback B. 2005.

Distinct hypermethylation profile of primary breast cancer is associated with sentinel lymph node metastasis. Clin Cancer Res 11:2156-2162. 10.1158/1078-0432.CCR-04-1810

Sparks AB, Morin PJ, Vogelstein B, and Kinzler KW. 1998. Mutational analysis of the APC/beta-catenin/Tcf pathway in colorectal cancer. Cancer Res 58:1130-1134.

Swellam M, Abdelmaksoud MD, Sayed Mahmoud M, Ramadan A, Abdel-Moneem W, and Hefny MM. 2015. Aberrant methylation of APC and RARbeta2 genes in breast cancer patients. IUBMB Life 67:61-68. 10.1002/iub.1346

Taback B, Giuliano AE, Lai R, Hansen N, Singer FR, Pantel K, and Hoon DS. 2006. Epigenetic analysis of body fluids and tumor tissues: application of a comprehensive molecular assessment for early-stage breast cancer patients. Ann N Y Acad Sci 1075:211-221. 10.1196/annals.1368.029 Torre LA, Bray F, Siegel RL, Ferlay J, Lortet-Tieulent J, and Jemal A. 2015. Global cancer statistics, 2012. CA Cancer J Clin 65:87-108. 10.3322/caac.21262

Van der Auwera I, Bovie C, Svensson C, Limame R, Trinh XB, van Dam P, Van Laere SJ, Van 436 Marck E, Vermeulen PB, and Dirix LY. 2009a. Quantitative assessment of DNA 
438

439

440

441

442

443

444

445

446

447

448

449

450

451

452

453

454

455

456

457

458

Biol Ther 8:2252-2259.

Van der Auwera I, Elst HJ, Van Laere SJ, Maes H, Huget P, van Dam P, Van Marck EA, Vermeulen PB, and Dirix LY. 2009b. The presence of circulating total DNA and methylated genes is associated with circulating tumour cells in blood from breast cancer patients. $B r J$ Cancer 100:1277-1286. 10.1038/sj.bjc.6605013

Van der Auwera I, Van Laere SJ, Van den Bosch SM, Van den Eynden GG, Trinh BX, van Dam PA, Colpaert CG, van Engeland M, Van Marck EA, Vermeulen PB, and Dirix LY. 2008. Aberrant methylation of the Adenomatous Polyposis Coli (APC) gene promoter is associated with the inflammatory breast cancer phenotype. $\mathrm{Br} J$ Cancer 99:1735-1742.

\subsection{8/sj.bjc.6604705}

Virmani AK, Rathi A, Sathyanarayana UG, Padar A, Huang CX, Cunnigham HT, Farinas AJ, Milchgrub S, Euhus DM, Gilcrease M, Herman J, Minna JD, and Gazdar AF. 2001. Aberrant methylation of the adenomatous polyposis coli (APC) gene promoter 1A in breast and lung carcinomas. Clin Cancer Res 7:1998-2004.

Wojdacz TK, Thestrup BB, Cold S, Overgaard J, and Hansen LL. 2011a. No difference in the frequency of locus-specific methylation in the peripheral blood DNA of women diagnosed with breast cancer and age-matched controls. Future Oncol 7:1451-1455. 10.2217/fon.11.123

Wojdacz TK, Thestrup BB, Overgaard J, and Hansen LL. 2011b. Methylation of cancer related genes in tumor and peripheral blood DNA from the same breast cancer patient as two independent events. Diagn Pathol 6:116. 10.1186/1746-1596-6-116

Zhang DP, Li XW, and Lang JH. 2015. Prognostic Value of beta-catenin Expression in Breast 
459 Cancer Patients: a Meta-analysis. Asian Pac J Cancer Prev 16:5625-5633.

460 Zhang JJ, Ouyang T, Wan WH, Xu GW, and Deng GR. 2007. [Detection and significance of 461 APC gene promoter hypermethylation in serum of breast cancer patients]. Ai Zheng 26:44-47.

462 Zmetakova I, Danihel L, Smolkova B, Mego M, Kajabova V, Krivulcik T, Rusnak I, Rychly B, 463 Danis D, Repiska V, Blasko P, Karaba M, Benca J, Pechan J, and Fridrichova I. 2013. 464 Evaluation of protein expression and DNA methylation profiles detected by pyrosequencing in 465 invasive breast cancer. Neoplasma 60:635-646. 10.4149/neo_2013_082 


\section{Figure 1 (on next page)}

Flow chart of the collection of studies for this meta-analysis. 
Records identified through The

Cochrane library, Pubmed, Web

of Science and Embase database searching $(\mathrm{n}=168)$
Manuscript to be reviewed

Additional records identified through other sources

$$
(\mathrm{n}=0)
$$

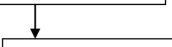

Records after duplicates removed

$(\mathrm{n}=167)$

Records excluded $(n=93)$

Records screened $(\mathrm{n}=74)$ Irrelevant to breast cancer $(\mathrm{n}=93)$

Full-text articles assessed for eligibility $(\mathrm{n}=31)$

PubMed 19, Web of

Science 10, Embase 2

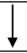

Full-text articles excluded $(n=43)$

No control group $(\mathrm{n}=21)$

Focus on tumor cell $(n=9)$

Review ( $\mathrm{n}=5$ )

Lack available data $(\mathrm{n}=8)$

Studies included in qualitative synthesis $(\mathrm{n}=35)$

included in quantitative synthesis

(meta-analysis)

$(\mathrm{n}=35)$ 
Figure 2 (on next page)

Forest plot of APC promoter methylation and breast cancer risk based on the random effects model.

The small squares and horizontal lines represent the $\mathrm{OR}$ and $95 \% \mathrm{Cl}$ of individual studies. If the $95 \% \mathrm{Cl}$ included 1, the difference in APC methylation between patients with breast cancer and controls was not significant. The centre of the diamond represents the combined treatment effect (calculated as a weighted average of individual ORs) and the horizontal tips represent the $95 \% \mathrm{Cl}$. OR represents the odds ratio. $95 \% \mathrm{Cl}$ represents the $95 \%$ confidence interval. 
breast cancer $\quad$ Control

Odds Ratio

Odds Ratio

Study or Subgroup

Events Gotal Events Total Weight

Brooks JD 2010

Chen KM 2011

Cho YH 2010

Dulaimi E 12004

Dulaimi E 22004

Fridrichova I 2015

Hoque MO 2006

Hoque MO 2009

Jeronimo C 2008

Jin Z 2001

Jing F 2010

Jung EJ 2013

Lee A 2004

Lewis CM 2005

Liu Z 2007

Martins AT 2011

Matuschek C 2010

Müller HM 2003

Pang JM 2014

Park SY 2011

Parrella P 2004

Prasad CP 12008

Prasad CP 22008

Rykova EY 2004

Shinozaki M 2005

Swellam M 2015

Taback B 2006

Van der A I 2009

Van der A I 12008

Van der A I 22008

Van der A I 2009

Virmani AK 2001

Wojdacz TK 2011

Zhang JJ 12007

Zhang JJ 22007

Total $(95 \% \mathrm{Cl})$

Total events

Heterogeneity: $\mathrm{Tau}^{2}=1.63 ; \mathrm{Chi}^{2}=106.88, \mathrm{df}=34(\mathrm{P}<0.00001) ; \mathrm{I}^{2}=68 \%$

Test for overall effect: $Z=7.95$ ( $P<0$. Repefferie)

\section{2}

$1212100.0 \%$
M-H, Random. $95 \%$ clpt to be reviputf.Random, $95 \% \mathrm{Cl}$

$\begin{array}{rrrrrr}1 & 49 & 6 & 142 & 3.0 \% & 0.47[0.06,4.02] \\ 12 & 17 & 1 & 10 & 2.8 \% & 21.60[2.13,218.58] \\ 21 & 40 & 12 & 27 & 4.4 \% & 1.38[0.52,3.68] \\ 15 & 34 & 0 & 6 & 2.1 \% & 10.33[0.54,197.97] \\ 10 & 34 & 0 & 20 & 2.2 \% & 17.57[0.97,318.41] \\ 144 & 206 & 0 & 9 & 2.2 \% & 43.93[2.52,766.48] \\ 8 & 47 & 0 & 38 & 2.2 \% & 16.57[0.92,297.10] \\ 56 & 112 & 3 & 32 & 4.1 \% & 9.67[2.78,33.57] \\ 55 & 66 & 10 & 12 & 3.6 \% & 1.00[0.19,5.21] \\ 18 & 50 & 0 & 21 & 2.2 \% & 24.48[1.40,427.99] \\ 14 & 50 & 0 & 50 & 2.2 \% & 40.12[2.32,694.45] \\ 19 & 60 & 0 & 60 & 2.2 \% & 56.86[3.34,968.06] \\ 14 & 33 & 0 & 19 & 2.2 \% & 29.00[1.61,520.88] \\ 15 & 27 & 14 & 55 & 4.5 \% & 3.66[1.39,9.67] \\ 28 & 76 & 0 & 76 & 2.3 \% & 89.91[5.36,1506.95] \\ 144 & 178 & 18 & 33 & 4.7 \% & 3.53[1.62,7.70] \\ 25 & 85 & 2 & 22 & 3.7 \% & 4.17[0.91,19.18] \\ 23 & 26 & 0 & 10 & 2.1 \% & 141.00[6.67,2980.83] \\ 39 & 80 & 0 & 15 & 2.2 \% & 29.51[1.71,509.92] \\ 72 & 85 & 7 & 30 & 4.4 \% & 18.20[6.48,51.07] \\ 15 & 54 & 1 & 10 & 3.0 \% & 3.46[0.40,29.72] \\ 6 & 32 & 0 & 5 & 2.1 \% & 2.70[0.13,55.26] \\ 11 & 50 & 0 & 50 & 2.2 \% & 29.41[1.68,514.42] \\ 4 & 10 & 0 & 6 & 2.0 \% & 9.00[0.40,203.30] \\ 74 & 151 & 0 & 10 & 2.2 \% & 20.19[1.16,350.67] \\ 113 & 121 & 0 & 66 & 2.2 \% & 1775.94[100.88,31265.40] \\ 1 & 33 & 0 & 10 & 1.9 \% & 0.97[0.04,25.64] \\ 60 & 100 & 0 & 9 & 2.2 \% & 28.38[1.61,501.35] \\ 28 & 51 & 3 & 27 & 4.0 \% & 9.74[2.60,36.49] \\ 53 & 54 & 7 & 9 & 2.5 \% & 15.14[1.21,189.44] \\ 15 & 78 & 1 & 19 & 3.0 \% & 4.29[0.53,34.68] \\ 19 & 45 & 3 & 28 & 4.0 \% & 6.09[1.60,23.16] \\ 24 & 180 & 13 & 108 & 4.7 \% & 1.12[0.55,2.31] \\ 38 & 84 & 0 & 84 & 2.3 \% & 139.92[8.40,2330.21] \\ 26 & 84 & 0 & 84 & 2.3 \% & 76.56[4.57,1281.31]\end{array}$

$9.97[5.66,17.57]$

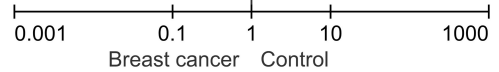




\section{Figure 3 (on next page)}

Sensitive analysis of pooled OR based on the random effects model.

The results were calculated by omitting each study in turn. The circles represent the individual studies in this meta-analysis. The two ends of the dotted lines represent the $95 \% \mathrm{Cl}$. OR represents the odds ratio. $95 \% \mathrm{Cl}$ represents the $95 \%$ confidence interval. 
PeerJ
Meta-analysis estimates, given named study is omitted | Lower CI Limit OEstimate

| Upper CI Limit

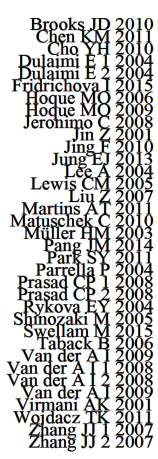

.

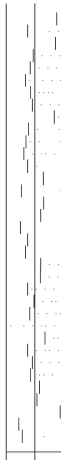


Figure 4 (on next page)

Publication bias analysis

(A) The funnel plot of APC methylation and breast cancer risk. The log of OR against the standard error of the log of the OR was plotted in this graph.(B) The Begg's plot of APC methylation and breast cancer risk. The circles represent the individual studies in this metaanalysis. The line in the centre represents the pooled OR. (C) The Begg's plot of publication bias after trim-and-fill analysis. The circles represent the included studies. The diamonds represent the presumed missing studies. OR represents the odds ratio. 


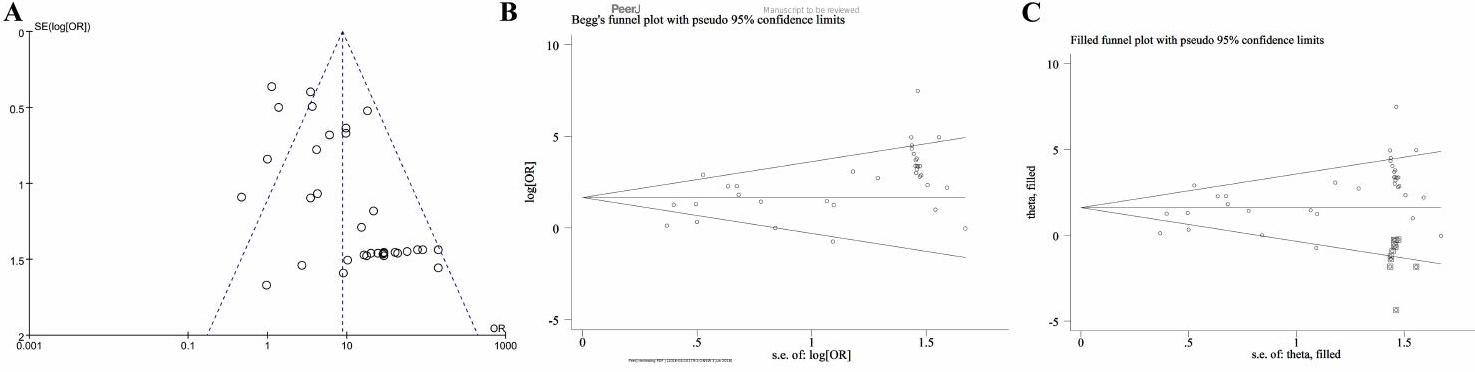


Table $\mathbf{1}$ (on next page)

General characteristics of the eligible studies. 
1 Table 1. General characteristics of eligible studies

\begin{tabular}{|c|c|c|c|c|c|c|c|c|c|c|}
\hline \multirow[t]{2}{*}{ Author } & \multirow[t]{2}{*}{ Year } & \multirow{2}{*}{$\begin{array}{l}\text { County/ } \\
\text { Region }\end{array}$} & \multirow[t]{2}{*}{ Method } & \multirow{2}{*}{$\begin{array}{l}\text { Sample } \\
\text { type }\end{array}$} & \multicolumn{2}{|l|}{$\mathbf{M} / \mathbf{N}$} & \multicolumn{2}{|c|}{ Stage $(M / N)$} & \multicolumn{2}{|c|}{ Grade(N/M) } \\
\hline & & & & & BC & Control & Early & Late & Low & High \\
\hline Brooks JD & 2010 & USA & QMSP & Serum & $1 / 49$ & $6 / 142$ & - & - & - & - \\
\hline Chen KM & 2011 & USA & MS-MLPA & FFT & $12 / 17$ & $1 / 10$ & - & - & - & - \\
\hline Cho YH & 2010 & USA & MethyLight & FFT & $21 / 40$ & $12 / 27$ & - & - & - & - \\
\hline Dulaimi E 1 & 2004 & USA & MSP & Surgery & $15 / 34$ & $0 / 6$ & $14 / 29$ & $1 / 5$ & $6 / 13$ & $9 / 18$ \\
\hline Dulaimi E 2 & & & & Serum & $10 / 34$ & $0 / 20$ & $9 / 29$ & $1 / 5$ & $4 / 13$ & $6 / 18$ \\
\hline Fridrichova I & 2015 & $\begin{array}{l}\text { Slovak } \\
\text { Republic }\end{array}$ & Pyro & FFPET & $144 / 206$ & $0 / 9$ & - & - & - & - \\
\hline Hoque MO & 2006 & West Africa & QMSP & Blood & $8 / 47$ & $0 / 38$ & - & - & - & - \\
\hline Hoque MO & 2009 & Italy & QMSP & FFPET & $56 / 112$ & $3 / 32$ & - & - & - & - \\
\hline Jeronimo C & 2008 & Portugal & QMSP & FFPET & $55 / 66$ & $10 / 12$ & - & - & - & - \\
\hline $\operatorname{Jin} Z$ & 2001 & Japan & MSP & Surgery & $18 / 50$ & $0 / 21$ & $13 / 36$ & $4 / 10$ & - & - \\
\hline Jing F & 2010 & China & MSP & Serum & $14 / 50$ & $0 / 50$ & - & - & $7 / 25$ & $12 / 25$ \\
\hline Jung EJ & 2013 & Korea & MS-MLPA & Surgery & $19 / 60$ & $0 / 60$ & $17 / 53$ & $2 / 7$ & $\begin{array}{l}13 / 4 \\
0\end{array}$ & $6 / 20$ \\
\hline Lee $\mathrm{A}$ & 2004 & Korea & MSP & NAF & $14 / 33$ & $0 / 19$ & $13 / 31$ & $1 / 2$ & - & - \\
\hline Lewis CM & 2005 & USA & MSP & NAF & $15 / 27$ & $14 / 55$ & - & - & - & - \\
\hline Liu Z & 2007 & China & MSP & Surgery & $28 / 76$ & $0 / 76$ & $15 / 54$ & $13 / 22$ & $\begin{array}{l}15 / 4 \\
8\end{array}$ & $13 / 28$ \\
\hline Martins AT & 2011 & Portugal & QMSP & NAF & $144 / 178$ & $18 / 33$ & - & - & - & - \\
\hline Matuschek C & 2010 & Germany & MethyLight & Blood & $25 / 85$ & $2 / 22$ & $5 / 42$ & $16 / 35$ & - & - \\
\hline Müller HM & 2003 & Austria & MethyLight & Serum & $23 / 26$ & $0 / 10$ & - & - & - & - \\
\hline Pang JM & 2014 & Australia & MS-HRM & FFPET & $39 / 80$ & $0 / 15$ & - & - & - & - \\
\hline Park SY & 2011 & South Korea & MethyLight & FFPET & $72 / 85$ & $7 / 30$ & - & - & $\begin{array}{l}13 / 3 \\
0\end{array}$ & $6 / 20$ \\
\hline Parrella P & 2004 & Italy & MSP & Tissue & $15 / 54$ & $1 / 10$ & - & - & - & - \\
\hline Prasad CP 1 & 2008 & India & MSP & Surgery & $6 / 32$ & $0 / 5$ & $2 / 19$ & $4 / 13$ & - & - \\
\hline Prasad CP 2 & & & & Serum & $11 / 50$ & $0 / 50$ & - & - & $4 / 28$ & $7 / 22$ \\
\hline Rykova EY & 2004 & Russia & MSP & Blood & $4 / 10$ & $0 / 6$ & - & - & - & - \\
\hline Shinozaki M & 2005 & USA & MSP & FFPET & $74 / 151$ & $0 / 10$ & - & - & - & - \\
\hline Swellam M & 2015 & Egypt & MSP & Serum & $113 / 121$ & $0 / 66$ & $81 / 86$ & $32 / 35$ & $\begin{array}{l}84 / 8 \\
9\end{array}$ & $29 / 32$ \\
\hline Taback B & 2006 & USA & QMSP & Blood & $1 / 33$ & $0 / 10$ & - & - & - & - \\
\hline Van der A I & 2009 & Belgium & QMSP & FFT & $60 / 100$ & $0 / 9$ & - & - & - & - \\
\hline Van der A I 1 & 2008 & Belgium & MSP & FFPET & $28 / 51$ & $3 / 27$ & - & - & - & - \\
\hline Van der A I 2 & & & QMSP & FFT & $53 / 54$ & $7 / 9$ & - & - & - & - \\
\hline Van der A I & 2009 & Belgium & QMSP & Blood & $15 / 78$ & $1 / 19$ & - & - & - & - \\
\hline Virmani AK & 2001 & USA & MSP & Surgery & $19 / 45$ & $3 / 28$ & - & - & - & - \\
\hline
\end{tabular}




\begin{tabular}{|l|l|l|l|l|l|l|l|l|l|l|}
\hline Wojdacz TK & 2011 & Denmark & MS-HRM & Blood & $24 / 180$ & $13 / 108$ & - & - & - & - \\
\hline Zhang JJ 1 & 2007 & China & MSP & Surgery & $38 / 84$ & $0 / 84$ & $30 / 66$ & $8 / 18$ & - & - \\
\hline Zhang JJ 2 & & & & Serum & $26 / 84$ & $0 / 10$ & $20 / 66$ & $6 / 18$ & - & - \\
\hline Total & & & & & $1220 /$ & $101 /$ & $219 /$ & $88 /$ & $146 /$ & $88 /$ \\
& & & & & 2482 & 1212 & 511 & 170 & 286 & 183 \\
\hline
\end{tabular}

2 MSP, methylation specific PCR. QMSP, quantitative real-time MSP. Pyro, Pyrosequencing. MS-HRM, methylation-sensitive

3 high-resolution melting analysis. FFPET, formalin fixed paraffin-embedded tissue. FFT, fresh frozen tissue. NAF, needle

4 aspirate fluid. MS-MLPA, methylation specific-multiplex ligation-dependent probe amplification. M, number of APC promoter 5 methylated patients. N, number of control.

6 


\section{Table 2 (on next page)}

Subgroup analysis for the association between APC promoter methylation and breast cancer. 
1 Table 2. Subgroup analysis for the relationship between APC promoter methylation and BC.

\begin{tabular}{|c|c|c|c|c|c|c|c|}
\hline \multirow[t]{2}{*}{ Subgroup } & \multirow[t]{2}{*}{ No } & \multirow{2}{*}{$\begin{array}{l}\text { BC } \\
M / N\end{array}$} & \multirow{2}{*}{$\begin{array}{l}\text { Control } \\
\mathbf{M} / \mathbf{N}\end{array}$} & \multirow[t]{2}{*}{ OR(95\%CI) } & \multicolumn{3}{|c|}{ Heterogeneity test } \\
\hline & & & & & $\mathbf{I}^{2}$ & $\mathbf{p}$ & $\mathrm{Chi}^{2}$ \\
\hline \multicolumn{8}{|l|}{ Sample types } \\
\hline Tissue & 19 & $772 / 1397$ & $47 / 480$ & $10.68[5.50,20.75]$ & $53 \%$ & 0.004 & 38.28 \\
\hline Blood or Serum & 13 & $275 / 847$ & $22 / 625$ & $11.79[3.02,46.06]$ & $80 \%$ & $<0.00001$ & 60.52 \\
\hline NAF & 3 & $173 / 238$ & $32 / 107$ & $3.95[2.10,7.42]$ & $6 \%$ & 0.34 & 2.13 \\
\hline \multicolumn{8}{|l|}{ Region } \\
\hline Asia & 10 & $246 / 604$ & $7 / 479$ & $27.21[13.51,54.81]$ & $0 \%$ & 0.69 & 6.47 \\
\hline Europe & 13 & $646 / 1200$ & $58 / 306$ & $5.50[2.69,11.22]$ & $60 \%$ & 0.003 & 30.00 \\
\hline North America & 9 & $168 / 430$ & $36 / 308$ & $3.79[1.70,8.45]$ & $42 \%$ & 0.09 & 13.83 \\
\hline Africa & 2 & $121 / 168$ & $0 / 104$ & $172.05[1.76,16792.96]$ & $80 \%$ & 0.02 & 5.07 \\
\hline Oceania & 1 & $39 / 80$ & $0 / 15$ & $29.51[1.71,509.92]$ & NA & NA & NA \\
\hline \multicolumn{8}{|l|}{ Methods } \\
\hline MSP & 17 & $448 / 986$ & $21 / 617$ & $18.18[7.96,41.52]$ & $54 \%$ & 0.004 & 35.03 \\
\hline QMSP & 9 & $393 / 717$ & $45 / 304$ & $3.92[1.77,8.70]$ & $40 \%$ & 0.10 & 13.27 \\
\hline MethyLight & 4 & $141 / 236$ & $21 / 89$ & $8.05[1.50,43.12]$ & $83 \%$ & 0.0006 & 17.29 \\
\hline MS-MLPA & 2 & $31 / 77$ & $1 / 70$ & $31.81[5.30,191.06]$ & $0 \%$ & 0.58 & 0.31 \\
\hline MS-HRM & 2 & $63 / 260$ & $13 / 123$ & $4.49[0.14,146.62]$ & $83 \%$ & 0.02 & 5.76 \\
\hline Pyro & 1 & $144 / 206$ & $0 / 9$ & $43.93[2.52,766.48]$ & NA & NA & NA \\
\hline
\end{tabular}

2 NAF, needle aspirate fluid. MSP, methylation specific PCR. QMSP, quantitative real-time MSP. Pyro, Pyrosequencing. MS-

3 MLPA, methylation specific-multiplex ligation-dependent probe amplification. MS-HRM, methylation-sensitive high-resolution 4 melting analysis. NA, not available. M, number of APC promoter methylated patients. N, number of control. 